\title{
Kernos
}

Revue internationale et pluridisciplinaire de religion grecque antique

12| 1999

Varia

\section{F. WULFF ALONSO, La Fortaleza asediada. Diosas, Héroes y Mujeres poderosas en el mito griego}

\section{François Jouan}

\section{OpenEdition \\ Journals}

\section{Édition électronique}

URL : http://journals.openedition.org/kernos/737

DOI : $10.4000 /$ kernos.737

ISSN : 2034-7871

\section{Éditeur}

Centre international d'étude de la religion grecque antique

\section{Édition imprimée}

Date de publication : 1 janvier 1999

Pagination : 311-313

ISSN : 0776-3824

\section{Référence électronique}

François Jouan, «F. wulfF ALonso, La Fortaleza asediada. Diosas, Héroes y Mujeres poderosas en el mito griego », Kernos [En ligne], 12 I 1999, mis en ligne le 13 avril 2011, consulté le 11 mars 2021. URL: http://journals.openedition.org/kernos/737 ; DOI : https://doi.org/10.4000/kernos.737 
On s'étonne un peu de ne voir exploitées dans aucune des contributions rassemblées ici les allusions du texte d'Euripide aux origines lydiennes du dieu. L'étude des textes et la comparaison des structures mythiques qui les soustendent sont les pistes empruntées par A. Moreau pour aborder le problème crucial de la signification initiatique du sacrifice de Penthée: dans la ligne de diverses interprétations récemment proposées du sacrifice dionysiaque ${ }^{1}$, l'auteur renouvelle la lecture du sacrifice du bouc-émissaire en le rapprochant d'autres sacrifices à caractère initiatique, tels ceux d'Actéon et d'Orphée, et conclut à l'existence d'une initiation manquée de Penthée dans les Bacchantes. Enfin, C. Jouanno s'est livrée à une étude de la chronique de J. Malalas, réinterprétation moralisante et politique de l'histoire de Dionysos et de Penthée.

$\grave{A}$ ces approches relativement classiques s'opposent les démarches de chercheurs venus d'autres horizons; l'iconographie, par exemple, permet à M. HalmTisserand de dresser un catalogue complet des scènes illustrant la mise à mort de Penthée et son démembrement par les femmes de Thèbes qui renouvelle sensiblement, sur ce point précis, le catalogue jadis établi par H. Philippart ${ }^{2}$. L. Beck Chauvard, comparant l'épiphanie dionysiaque des Baccbantes à l'épiphanie du dieu auprès d'Ariane, souligne les éléments communs aux deux apparitions, mais aussi leurs profondes différences. Dans une optique davantage philosophique, S. Vilatte a tenté de montrer, en une comparaison éclairante, dans quelle mesure le dionysisme peut constituer une clé de lecture du socratisme puisque c'est un fidèle du dionysisme qui se lit en filigrane du personnage de Socrate dans le Phèdre, fidèle tout épris d'un dionysisme sage et chaste tel que le prônent les bacchantes. Dans Dionysos et le crucifié, B. Fricker met en évidence les phénomènes présidant à la resémantisation des symboles du dionysisme et du christianisme que sont le thyrse et la croix. La place des Bacchantes comme pièce destinée au théâtre est abordée par J. Chemouni; et ce sont les récits comme mode d'expression qui sont mis en exergue dans l'article de M. Lacore; enfin, B. Deforge montre combien, en suggérant le démembrement de Penthée par le biais du récit, Euripide va loin dans la «monstration » de la mort.

Parce qu'elle touche aux universaux constitutifs de la personnalité humaine, cette tragédie qui met aux prises un dieu et un roi, qu'unissent aussi des liens de parenté, mais surtout une mère et son fils, est passible d'une interprétation psychanalytique; M.-J. Bataille, dans «Pulsions " de vie et "pulsions » de mort dans les Bacchantes, en décortique les ressorts intimes, en proposant du fatal affrontement une lecture freudienne. De trop nombreuses coquilles émaillent cependant assez tristement plusieurs articles de ce livre enthousiasmant qui révèle quel bénéfice un texte aussi connu que les Bacchantes peut retirer d'une approche interdisciplinaire.

Isabelle Tassignon (Université de Liège)

Fernando Wulf f Alonso, La Fortaleza asediada. Diosas, Héroes y Mujeres poderosas en el mito griego, Salamanca, Ediciones Universidad, 1997. 1 vol. $17 \times 24 \mathrm{~cm} .357$ p. (Acta Salmanticensia. Estudios bistóricos $\&$ geogräficos, 105). ISBN : 84-7481-813-6.

1 D. OBbINk, Dionysus Poured Out: Ancient and Modern Tbeories of Sacrifice and Cultural Formation, dans T.H. Carpenter et C.A. Faraone (éds), Masks of Dionysus, IthacaLondres, 1993, p. 65-88; R. SEAFORD, Reciprocity and Ritual, Oxford, 1994.

2 H. Philippart, L'iconograpbie des « Bacchantes » d'Euripide, Paris, 1930. 
La métaphore de la «forteresse assiégée » qui donne son titre à l'ouvrage illustre la double domination qui constitue la loi quasi invariable de toutes les sociétés, celle de l'élément divin sur l'élément humain, et celle du sexe masculin sur le sexe féminin. Mais dans les deux cas, il ne s'agit pas d'une domination sereine, car elle est vécue dans la crainte de tentatives de renversement du pouvoir, menées par les hommes contre les dieux, et surtout par la partie féminine - déesses ou simples femmes - contre le pouvoir mâle. C'est du moins ainsi, selon l'A., que la réalité de ce combat potentiel se vérifie à travers les mille légendes de la mythologie grecque. Les temps mythiques sont définis par lui comme des temps de désordre et de violence, qui permettent des contacts physiques entre dieux et hommes, et en particulier des rencontres sexuelles qui sont autant de violations des normes. Passe pour le cas où une mortelle est séduite par un dieu, puisqu'il traduit la double supériorité du divin et du masculin. L'anomalie est beaucoup plus grave quand une déesse se soumet au désir d'un amant humain, comme Circé et Calypso dans l'Odyssée, ou même quand une «femme de pouvoir » impose sa domination à un héros, comme Omphale avec Héraclès.

L'étude est constituée de dix chapitres, chacun se subdivisant en séquences assez courtes, d'une à cinq pages en moyenne. Les trois premiers sont consacrés à un examen approfondi des deux épisodes odysséens, dont l'A. nous dit qu'ils ont constitué le point de départ de sa réflexion. Assez curieusement, c'est dans le chapitre du milieu que sont exposés les principes généraux qui conduiront la recherche. Après un retour à Circé et à Calypso, l'A. cite d'autres divinités qui se sont unies à un mortel, comme Thétis et Déméter, puis il passe à des monstres féminins de l'Odyssée, Sirènes, Scylla, Charybde, figures non dépourvues à son avis de connotations sexuelles. Le chapitre IV oppose le cas d'une déesse qui ne dissimule pas sa nature à son amant humain, celui d'Aphrodite avec Anchise (épisode dont l'étude est intéressante, mais un peu rapide), à la situation qui concerne des divinités dissimulées ou dégradées sous des apparences mortelles, comme Pasiphaé. Dans la légende des deux héros Minos et Thésée apparaissent des femmes dangereuses, qu'ils affrontent avec des sorts divers : Pasiphaé, Procris, Ariane, Hélène... Le chapitre $V$, à notre sens un des meilleurs du livre, est consacré à la captivité d'Héraclès chez Omphale, qui marque le point extrême de l'abaissement du héros. C'est l'occasion d'évoquer quelques cas de captivité de dieux chez des mortels, comme Apollon chez Admète, Apollon et Poséidon chez Laomédon.

Le chapitre VI passe en revue un certain nombre de figures féminines, de déesses ou de femmes, détentrices d'un pouvoir fort et dangereux pour l'autre sexe : Io, Sémélé, Europe, Alcmène. On revient encore à Omphale, pour s'attarder sur l'Arétè des Phéaciens et sur les Danaïdes. Puis le défilé s'accélère, énumérant les responsables et souvent aussi les victimes féminines de catastrophes: Alcmène, Cométo, Médousa, fille de Ninos, Antigone et Astydamie dans la légende de Pélée, Sthénébée-Anteia dans celle de Bellérophon, les Proetides, Hippodamie, Atalante, Althée, Déjanire et Léda.

Le champ se resserre au chapitre suivant avec l'examen des grandes héroïnes des cycles thébain et troyen, Eriphyle, Hypsipyle, les femmes d'Alcméon, Hélène, Clytemnestre, Pénélope. C'est au «fils de la déesse », Achille, qu'est consacré le chapitre suivant, mais il est plus longuement question du «plan de Zeus » qui, pour l'auteur, encadre de façon rigoureuse le déroulement de l'lliade, le roi des dieux manipulant les êtres divins et humains à ses fins propres. Ce chapitre insiste aussi sur le rôle ambigu dans le poème des «objets divins » (conçus au sens le plus large, puisqu'ils comprennent aussi les murailles de Troie). Avec le 
chapitre IX, on revient à l'Odyssée et à Ulysse, dont les aventures et la conduite, elles aussi, sont des éléments du «plan de Zeus». Ces deux chapitres précédant la conclusion développent l'idée que la fin de Troie et de Thèbes, relativement proches dans le temps mythique, marquent toutes deux la fin du monde des héros, d'un monde où dieux et hommes pouvaient se mêler physiquement les uns aux autres, pour le meilleur et pour le pire. Ensuite commence le temps de l'histoire, qui traduit la séparation définitive des deux races : désormais, elles ne pourront plus se rejoindre que par le truchement de la religion et des rites, éliminant ainsi les dangers les plus graves de la période antérieure et les risques de violation de l'ordre hiérarchique, seul garant du bon développement des sociétés. Le dernier chapitre, sous le titre: "Histoire, mythe, sexe », rassemble les conclusions de l'étude, dont nous avons, chemin faisant, indiqué les principaux points forts.

La matière brassée par cet ouvrage est, on le voit, considérable. On ne peut pourtant dire qu'elle épuise le sujet, car quel est le mythe grec qui, d'une façon ou d'une autre, ne traduit pas un affrontement entre dieux et hommes, entre représentants de l'un et l'autre sexe, ou une transgression des hiérarchies sociales? Mais on voit bien la vanité qu'il y aurait à dresser un tel catalogue, et certaines parties du livre en montrent le risque : ainsi pour les chapitres VI et VII, où l'abondance des exemples cités ne permet qu'un survol agrémenté d'appréciations rapides, pour des légendes aussi chargées de sens que celles d'Io, des Danaïdes, de Sthénébée, des Proetides, de Jocaste, d'Hypsipyle ou de Médée. Il aurait été plus fructueux, à mon sens, de limiter les exemples choisis et d'approfondir l'examen des plus significatifs, ce qui a été bien fait pour Omphale et aurait pu l'être mieux pour Aphrodite et Anchise. D'autre part, la marche à la fois sinueuse et sautillante de l'exposé, avec ses écarts et ses retours (plusieurs fois marqués par la formule : «Revenons à... ») évoque plus une promenade à travers les mythes qu'une ferme avancée vers un but. Ici et là figurent des légendes vraiment marginales (ainsi p. 125-130 sur les exploits d'Héraclès lors de son séjour chez Omphale). Les idées générales, étant en définitive peu nombreuses, reparais sent avec une périodicité excessive, qui ralentit l'exposé.

Ces quelques remarques, qui touchent à la méthode, n'empêchent nullement de reconnaitre que l'auteur manifeste une connaissance approfondie des mythes grecs et de la "littérature " qui s'y rattache : témoin la copieuse bibliographie qui termine l'ouvrage et qui est utilisée à bon escient dans les notes de bas de page. Celles-ci sont sobres, mais le plus souvent elles mentionnent les études les plus valables sur le point en question. La forme est extrêmement soignée. L'auteur s'est visiblement investi tout entier dans son sujet, il s'est constitué une doctrine contestable dans certains cas, mais en partie originale, et il a rassemblé une somme documentaire dont la consultation sera souvent profitable.

François Jouan

(Université de Paris X - Nanterre)

José Carlos Bermejo Barrera, Francisco J. GonzÁlez García, Susana ReBoreda Morillo, Los origenes de la mitología griega, Madrid, Ediciones Akal, 1996, 429 p. (Akal Universitaria. Serie Interdisciplinar). ISBN : 84-4600580-8.

J.C. Bermejo Barrera et ses deux collaborateurs convient le lecteur à une réflexion sur les origines de la religion grecque privilégiant l'approche socioanthropologique des textes. Après un premier chapitre méthodologique qui fait l'état de la question de la religion créto-mycénienne et des diverses interpréta tions qui en furent données depuis les travaux d'Evans jusqu'à ceux de Renfrew et de Pötscher, J.C. Bermejo Barrera consacre un chapitre aux unions matrimo- 